\title{
ON ALGEBRAS OF BANACH ALGEBRA-VALUED BOUNDED CONTINUOUS FUNCTIONS
}

\author{
HUGO ARIZMENDI-PEIMBERT, ANGEL CARRILLO-HOYO \\ AND ALEJANDRA GARCÍA-GARCÍA
}

\begin{abstract}
Let $X$ be a completely regular Hausdorff space. We denote by $C(X, A)$ the algebra of all continuous functions on $X$ with values in a complex commutative unital Banach algebra $A$. Let $C_{b}(X, A)$ be its subalgebra consisting of all bounded continuous functions and endowed with the uniform norm. In this paper, we give conditions equivalent to the density of a natural continuous image of $X \times \mathcal{M}(A)$ in the maximal ideal space of $C_{b}(X, A)$.
\end{abstract}

1. Introduction. Throughout this paper, $X$ will denote a completely regular Hausdorff space, $A$ a complex commutative unital Banach algebra with norm $\|\cdot\|$ and unit element $e$ and $G(A)$ the set of invertible elements of $A$. We may assume that $\|e\|=1$. We shall use the following notation for various function spaces:

$C(X, A)$ is the unital algebra of all continuous functions on $X$ with values in $A$, with pointwise operations and unit element the function on $X$ identically equal to $e$ and which will be denoted simply by $e$.

$C_{b}(X, A)$ is the subalgebra of $C(X, A)$ of all bounded continuous functions, provided with the uniform norm $\|\cdot\|_{\infty}$ given by $\|f\|_{\infty}=$ $\sup _{x \in X}\|f(x)\|$.

When $A$ is the complex field $\mathbb{C}$, then we shall write $C(X)$ and $C_{b}(X)$ instead of $C(X, \mathbb{C})$ and $C_{b}(X, \mathbb{C})$, respectively.

$C_{p}(X, A)$ is the subalgebra of $C_{b}(X, A)$ of all continuous functions $f$ such that the closure of its range in $A$, namely cl $(f(X))$, is compact in $A$.

2010 AMS Mathematics subject classification. Primary 46E40, 46H05, 46J10.

Keywords and phrases. Banach algebras, vector-valued bounded continuous functions, maximal ideal space.

Received by the editors on October 14, 2013, and in revised form on June 4, 2014. 
It is easy to see that $C_{b}(X, A)$ and $C_{p}(X, A)$ are Banach algebras. In general, $C_{p}(X, A)$ is a proper subalgebra of $C_{b}(X, A)$ as the next example shows. Take $X=\mathbb{N}$ endowed with the discrete topology and $A=C([0,1])$ with the uniform norm. Let $f: X \rightarrow A$ be the function given by $f(n)(0)=f(n)(1)=1, f(n)(1-1 / n)=1 / n$ and $f(n)$ is linear elsewhere in $[0,1]$. Then $f \in C_{b}(X, A) \backslash C_{p}(X, A)$, since the sequence $(f(n))$ has no uniformly convergent subsequence in $C([0,1])$.

Necessary and sufficient conditions for the equality of the latter algebras are given in the next easily proven result.

Proposition 1.1. The following assertions are equivalent:

(i) $C_{b}(X, A)=C_{p}(X, A)$.

(ii) If $f \in C_{b}(X, A)$ and $f(X) \subset G(A)$, then $c l(f(X))$ is compact.

(iii) For every $f \in C_{b}(X, A)$, there exist $\lambda_{1}, \lambda_{2} \in \mathbb{C}$, with $\lambda_{1} \neq 0$, such that $\lambda_{1} f+\lambda_{2} e \in C_{p}(X, A)$.

For every $f \in C_{p}(X, A)$, there exists a unique extension $f_{\beta}$ of $f$ to the Stone-Čech compactification $\beta X$ of $X$.

Let $B$ be any complex commutative unital algebra. We denote by $\mathfrak{M}^{\#}(B)$ the set of all non-zero multiplicative linear functionals on $B$, provided with the weak star topology $w^{*}$. When $B$ is a topological algebra, $\mathfrak{M}(B)$ denotes the topological subspace of $\mathfrak{M}^{\#}(B)$ consisting of all non-zero multiplicative continuous linear functionals on $B$. For $b \in B$, its Gelfand transform $\widehat{b}$ is given by $\widehat{b}(\varphi)=\varphi(b)$, for $\varphi \in \mathfrak{M}^{\#}(B)$. The set $\mathfrak{M}(B)$ is called the maximal ideal space of $B$ and it coincides with $\mathfrak{M}^{\#}(B)$ if $B$ is a Banach algebra.

There are several papers in which $\mathfrak{M}^{\#}(B)$ or $\mathfrak{M}(B)$ is characterized when $B$ is a function algebra. Well-known results are: $\mathfrak{M}^{\#}(C(X))=$ $X$ if $X$ is a realcompact space ([5, page 3609 , Theorem 1$])$ and $\mathfrak{M}\left(C_{b}(X)\right)=\beta(X)$ if $X$ is a completely regular Hausdorff space $([\mathbf{1 1}$, page 123 , Theorem (3.2.11)]).

Along these lines, Dierolf, Schröder and Wengenroth proved in [3, page 54, Theorem 1], the formula $\mathfrak{M}^{\#}(C(X, E))=X \times \mathfrak{M}^{\#}(E)$ for a (completely regular Hausdorff) realcompact space $X$ and a metrizable topological algebra $E$. Under the same assumption on $X$ this formula was previously proved in [8, page 371, Theorem 5 (a)] by Hery 
supposing that $E$ is a unital commutative topological $Q$-algebra with continuous inversion and either $\mathfrak{M}(E)$ is locally equicontinuous or $X$ is discrete.

Concerning the maximal ideal spaces of functions algebras, Hausner in [7, page 248, Theorem], Dietrich in [4, page 207, Theorem 4] and Kahn in $([\mathbf{9}$, page 89 , Theorem 5.2.4]) proved that $\mathfrak{M}(C(X, E))=$ $X \times \mathfrak{M}(E)$. In the first of these works $X$ is a compact Hausdorff space and $E$ is a unital complex commutative Banach algebra. In the second one, $X$ is any completely regular $k$-space and $E$ is a unital complete locally convex algebra such that $\mathfrak{M}(E)$ is locally equicontinuous. In Kahn's, $X$ is a completely regular space of finite covering dimension and $E$ is a unital topological algebra with non-trivial dual and such that $\mathfrak{M}(E)$ is locally equicontinuous. In all these papers $C(X, E)$ carries the compact-open topology. Using any of these results or $[\mathbf{1}$, page 314 , Corollary 6], the equality $\mathfrak{M}\left(C_{p}(X, A)\right)=\beta X \times \mathfrak{M}(A)$, which is a particular case of $[\mathbf{8}$, page 369 , Corollary 2 (a)], is easily obtained in Proposition 2.1 under our general hypothesis on $X$ and $A$.

In contrast, little is known in general about the maximal ideal space of $C_{b}(X, A)$. Govaerts showed in [6, page 156, Counterexample 1] that $\mathfrak{M}\left(C_{b}(X, A)\right)=\beta X \times \mathfrak{M}(A)$ is false in general, and Kahn proved in [9, page 89, Corollary 5.2.3] that $\mathfrak{M}\left(C_{b}(X, E)\right)=X \times \mathfrak{M}(E)$, where $C_{b}(X, E)$ is endowed with the strict topology for any completely regular space $X$ of finite covering dimension and a unital topological algebra $E$ with non-trivial dual for which $\mathfrak{M}(E)$ is locally equicontinuous. The notion of the strict topology on $C_{b}(X, E)$ was first introduced by Buck in [2, page 97 , Definition] in the case of $X$ locally compact and $E$ locally convex.

Here we study $\mathfrak{M}\left(C_{b}(X, A)\right)$. We define a natural continuous transformation $T$ from $X \times \mathfrak{M}(A)$, with the product topology, into $\mathfrak{M}\left(C_{b}(X, A)\right)$. Therefore, each function $f \in C_{b}(X, A)$ has its proper Gelfand transform $\widehat{f} \in C\left(\mathfrak{M}\left(C_{b}(X, A)\right)\right)$ and also another Gelfand transform $\tilde{f}=\widehat{f} \circ T$ belonging to $C_{b}(X \times \mathfrak{M}(A))$. We prove that the transformation $f \rightarrow \widetilde{f}$ is a continuous homomorphism.

Let $A$ be a complex completely symmetric algebra, i.e., a complex commutative unital Banach algebra with involution * satisfying $\|a\|=$ $\left\|a^{*}\right\|$ and $F\left(a^{*}\right)=\overline{F(a)}$ (the complex conjugate of $F(a)$ ) for all $a \in A$ 
and $F \in \mathfrak{M}(A)$. We show that property " $f \in C_{b}(X, A)$ is invertible if $\tilde{f}$ is invertible" is equivalent to " $T(X \times \mathfrak{M}(A))$ is dense in $\mathfrak{M}\left(C_{b}(X, A)\right)$."

We do not know if these two properties are still equivalent if $A$ is not assumed as above, but we exhibit an example, orally proposed by $\mathrm{V}$. Müller, in which $A$ is a complex completely symmetric algebra and nevertheless there exists $f \in C_{b}(X, A)$ such that $\tilde{f}$ is invertible and $f$ is not. Therefore even for completely symmetric algebras, the set $\mathfrak{M}\left(C_{b}(X, A)\right)$ is in general larger than the $w^{*}$-closure $c l_{w^{*}}(T(X \times$ $\mathfrak{M}(A)))$ of $T(X \times \mathfrak{M}(A))$.

Any $C^{*}$-algebra is an example of a completely symmetric algebra ([10, page 233, Corollary 4]), but here we are not going to assume that the involution on $A$ satisfies $\left\|a a^{*}\right\|=\|a\|^{2}$, not even the weaker condition $\left\|a a^{*}\right\|=\|a\|\left\|a^{*}\right\|$, for $a \in A$.

2. Results. In this section, we define a natural continuous transformation $T$ from $X \times \mathfrak{M}(A)$, with the product topology, into $\mathfrak{M}\left(C_{b}(X, A)\right)$ and through it and the classical Gelfand transform $\widehat{f}$ for $f \in C_{b}(X, A)$, we introduce the Gelfand transform $\widetilde{f}$ with respect to $X \times \mathfrak{M}(A)$. Using $T$ and $\widetilde{f}$, we shall state and prove almost all the results. In order to avoid confusion on the scope of these, we recall that we are assuming that $X$ is a completely regular Hausdorff space and $A$ is a complex commutative unital Banach algebra. From Lemma 2.5 on, $A$ is a complex completely symmetric algebra with continuous involution.

Proposition 2.1. The function $H: C_{p}(X, A) \rightarrow C(\beta X, A)$, with $H(f)=f_{\beta}$ is an isometric isomorphism of algebra and $\mathfrak{M}\left(C_{p}(X, A)\right)=$ $\beta X \times \mathfrak{M}(A)$.

Proof. It is readily seen that $H$ is a bijective homomorphism of algebras. We also have that $\|f\|_{\infty}=\left\|f_{\beta}\right\|_{\infty}$, since $X$ is dense in $\beta X$ and then $H$ is an isometry. Thus, $\mathfrak{M}\left(C_{p}(X, A)\right)=\mathfrak{M}(C(\beta X, A))$. Since $\mathfrak{M}(C(\beta X, A))=\beta X \times \mathfrak{M}(A)$, the result follows.

Proposition 2.2. There exists a continuous mapping $T$ from $X \times$ $\mathfrak{M}(A)$ into $\mathfrak{M}\left(C_{b}(X, A)\right)$, given by $T(x, F)=T_{(x, F)}$, where

$$
T_{(x, F)}(f)=F(f(x))=\widehat{f(x)}(F),
$$


for every $f \in C_{b}(X, A)$ and $\widehat{f(x)}$ is the Gelfand transform of $f(x)$. This mapping $T$ has a continuous extension $T_{\beta}$ to $\beta(X \times \mathfrak{M}(A))$.

Proof. It is clear that $T_{(x, F)} \in \mathfrak{M}\left(C_{b}(X, A)\right)$. Given the $w^{*}$-neighborhood $U=V\left(T_{(x, F)}, f_{1}, \ldots, f_{n}, \epsilon\right)$ of $T_{(x, F)}$ take the $w^{*}$ - neighborhood $W=V\left(F, f_{1}(x), \ldots, f_{n}(x), \epsilon / 2\right)$ of $F$ and a neighborhood $V(x)$ of $x$ satisfying $\left\|f_{i}(x)-f_{i}(y)\right\|<\epsilon / 2$ if $y \in V(x)$ and $1 \leq i \leq n$. Then, for $(y, G) \in V(x) \times W$, we have that $T_{(y, G)} \in U$.

Since $\mathfrak{M}\left(C_{b}(X, A)\right)$ is compact, then $T$ has a continuous extension $T_{\beta}$ to $\beta(X \times \mathfrak{M}(A))$.

Corollary 2.3. $T_{\beta}(\beta(X \times \mathfrak{M}(A)))=\operatorname{cl}_{w^{*}}(T(X \times \mathfrak{M}(A)))$.

Proof. Since $T_{\beta}$ is continuous and $X \times \mathfrak{M}(A)$ is dense in $\beta(X \times$ $\mathfrak{M}(A))$, we get that $T_{\beta}(\beta(X \times \mathfrak{M}(A))) \subset \operatorname{cl}_{w^{*}}(T(X \times \mathfrak{M}(A)))$. But $T_{\beta}(\beta(X \times \mathfrak{M}(A)))$, being weak*- compact, contains the weak*-closure of $T(X \times \mathfrak{M}(A))$.

Taking $f \in C_{b}(X, A)$, we define its Gelfand's transform $\tilde{f}$ with respect to $X \times \mathfrak{M}(A)$ as $\widetilde{f}=\widehat{f} \circ T$, i.e.,

$$
\tilde{f}(x, F)=F(f(x)),
$$

for $(x, F) \in X \times \mathfrak{M}(A)$. Therefore, $\tilde{f} \in C_{b}(X \times \mathfrak{M}(A))$ and $\|\widetilde{f}\|_{\infty} \leq$ $\|f\|_{\infty}$.

The mapping $f \rightarrow \widetilde{f}$ is a continuous homomorphism from $C_{b}(X, A)$ into $C_{b}(X \times \mathfrak{M}(A))$. Thus, if $f$ is invertible in $C_{b}(X, A)$, then $\tilde{f}$ is invertible in $C_{b}(X \times \mathfrak{M}(A))$.

The function $\tilde{f}$ is invertible in the algebra $C_{b}(X \times \mathfrak{M}(A))$ if and only if $\tilde{f}$ is bounded away from zero, i.e., $|F(f(x))|>\epsilon$ for some $\epsilon>0$ and all $(x, F) \in X \times \mathfrak{M}(A)$. In particular, $f$ is invertible in $C(X, A)$ if $\tilde{f}$ is invertible.

Theorem 2.4. For the following four assertions we have that: (i) implies (ii); (ii) implies (iv); and (ii) and (iii) are equivalent to each other. 
(i) If $f_{1}, \ldots, f_{n} \in C_{b}(X, A)$ and $\epsilon>0$ are such that, for every $(x, F) \in X \times \mathfrak{M}(A)$, there exist $1 \leq i \leq n$ for which $\left|\widetilde{f}_{i}(x, F)\right|>\epsilon$, then there exist $g_{1}, \ldots, g_{n} \in C_{b}(X, A)$ satisfying $f_{1} g_{1}+\cdots+$ $f_{n} g_{n}=e$.

(ii) If $f \in C_{b}(X, A)$ and $\tilde{f}$ is invertible, then $f$ is invertible.

(iii) If $f \in C_{b}(X, A)$ and there exists $\epsilon>0$ such that $\|f(x)-y\|>\epsilon$ for all $x \in X$ and $y \in A \backslash G(A)$, then $f$ is invertible.

(iv) If $f \in C_{b}(X, A)$ and

$$
\sup \{|\tilde{f}(x, F)|:(x, F) \in X \times \mathfrak{M}(A)\}<1,
$$

then $e-f$ is invertible.

Proof. Obviously, (i) implies (ii) and (ii) implies (iv).

(ii) $\Rightarrow$ (iii). Assume that there exists $\epsilon>0$ such that $\|f(x)-y\|>\epsilon$ for all $x \in X$ and $y \in A \backslash G(A)$. Put $y=f(x)-F(f(x)) e$ for $x \in X$ and $F \in \mathfrak{M}(A)$. We have that $y \notin G(A)$ and $|\tilde{f}(x, F)|=|F(f(x))|=$ $\|f(x)-y\|>\epsilon$, then $\widetilde{f}$ is invertible and, by (ii), $f$ is invertible.

(iii) $\Rightarrow$ (ii). Take $f \in C_{b}(X, A)$, and suppose $\tilde{f}$ is invertible. There exists an $\epsilon>0$ such that $|\widetilde{f}(x, F)|>\epsilon$ for all $(x, F) \in X \times \mathfrak{M}(A)$. Given $x \in X$ and $y \in A \backslash G(A)$, choose $F \in \mathfrak{M}(A)$ such that $F(y)=0$ and put $y=f(x)-F(f(x)) e$. Then, $\|f(x)-y\|=|\tilde{f}(x, F)|>\epsilon$; hence by (iii), $f$ is invertible.

In the rest of this section we shall assume that $A$ is a complex completely symmetric algebra with continuous involution *.

Lemma 2.5. For every $f \in C_{b}(X, A)$, there exists a $g \in C_{b}(X, A)$ such that $\widetilde{g}(x, F)$ is the complex conjugate $\widetilde{\widetilde{f}(x, F)}$ of $\widetilde{f}(x, F)$ for each $(x, F) \in X \times \mathfrak{M}(A)$. Furthermore, we have $|\widetilde{f}|^{2}=\widetilde{f g}$.

Proof. If $f \in C_{b}(X, A)$, then the function $g$ defined by $g(x)=f(x)^{*}$ belongs to $C_{b}(X, A)$ because the involution is a continuous function. Then, we have

$$
\widetilde{g}(x, F)=F\left(f(x)^{*}\right)=\overline{\widetilde{f}(x, F)}
$$


and

$$
\widetilde{f g}(x, F)=F\left(f(x) f(x)^{*}\right)=|\widetilde{f}(x, F)|^{2},
$$

for all $(x, F) \in X \times \mathfrak{M}(A)$.

Theorem 2.6. Assertions (i)-(iv) in Theorem 2.4 are all equivalent.

Proof.

(iv) $\Rightarrow$ (ii). Take $f \in C_{b}(X, A)$, and suppose that $\widetilde{f}$ is invertible. Then, $\tilde{f}$ is bounded away from zero. Take $g$ as in Lemma 2.5, and set $M=\sup |\widetilde{f}(x, F)|^{2}$ and $\left.N=\sup \mid e-\widetilde{(1 / M}\right) f g(x, F) \mid$, where the suprema are taken over all points $(x, F)$ in $X \times \mathfrak{M}(A)$. Since $N=\left.\sup |1-(1 / M)| \widetilde{f}(x, F)\right|^{2} \mid<1$, we have by (iv) that $(1 / M) f g$ is invertible and then (ii) holds.

(ii) $\Rightarrow$ (i). Suppose $f_{1}, \ldots, f_{n} \in C_{b}(X, A)$ and $\epsilon>0$ are as in (i). Let $g_{i} \in C_{b}(X, A)$ be such that $\left|\widetilde{f}_{i}\right|^{2}=\widetilde{f_{i} g_{i}}$ for every $i=1,2, \ldots, n$. For $(x, F) \in X \times \mathfrak{M}(A)$ we have that $\sum_{i=1}^{n}\left|\widetilde{f}_{i}(x, F)\right|^{2}=\sum_{i=1}^{n} \widetilde{f_{i} g_{i}}(x, F)=$ $\widetilde{\sum_{i=1}^{n} f_{i}} g_{i}(x, F)>\epsilon$. Thus, $\widetilde{\sum_{i=1}^{n} f_{i}} g_{i}$ is invertible in $C_{b}(X \times \mathfrak{M}(A))$. By (ii), $\sum_{i=1}^{n} f_{i} g_{i}$ is invertible; therefore, there exists $h \in C_{b}(X, A)$ such that $\sum_{i=1}^{n} f_{i} g_{i} h=e$, that is, (i) holds.

Proposition 2.7. If $T(X \times \mathfrak{M}(A))$ is not dense in $\mathfrak{M}\left(C_{b}(X, A)\right)$, then there exists an $f \in C_{b}(X, A)$ such that $\tilde{f}$ is invertible and $f$ is not.

Proof. Let us assume that $T(X \times \mathfrak{M}(A))$ is not dense in $\mathfrak{M}\left(C_{b}(X, A)\right)$, and take $G \in \mathfrak{M}\left(C_{b}(X, A)\right) \backslash \mathrm{cl}_{w^{*}}(T(X \times \mathfrak{M}(A)))$. Then, there exist $f_{1}, \ldots, f_{n} \in C_{b}(X, A)$ and $\epsilon>0$ such that, for each $(x, F) \in X \times \mathfrak{M}(A)$, there is a $1 \leq i \leq n$ such that $\left|G\left(f_{i}\right)-F\left(f_{i}(x)\right)\right|>\epsilon$. Put $g_{i}=$ $f_{i}-G\left(f_{i}\right) e$, and take $h_{i} \in C_{b}(X, A)$ such that $\widetilde{h_{i}}(x, F)=\overline{\widetilde{g}_{i}(x, F)}$ for $1 \leq i \leq n$ and $(x, F) \in X \times \mathfrak{M}(A)$. Then, for each $(x, F) \in X \times \mathfrak{M}(A)$, $\left|\widetilde{g}_{i}(x, F)\right|>\epsilon$ for some $1 \leq i \leq n$ and $G\left(g_{i}\right)=0$ for all $1 \leq i \leq n$.

Take

$$
f=\sum_{i=1}^{n} g_{i} h_{i}
$$


Then $G(f)=0$ and

$$
|\widetilde{f}(x, F)|=\sum_{i=1}^{n}\left|\widetilde{g}_{i}(x, F)\right|^{2}>\epsilon
$$

for all $(X, F) \in X \times \mathfrak{M}(A)$. Therefore, $f$ is not invertible and $\tilde{f}$ is invertible.

Theorem 2.8. Assertions (i)-(iv) of Theorem 2.4 are all equivalent to the following:

(v) $T(X \times \mathfrak{M}(A))$ is dense in $\mathfrak{M}\left(C_{b}(X, A)\right)$.

Proof. From Proposition 2.7, (ii) implies (v). On the other hand, let us assume that $T(X \times \mathfrak{M}(A))$ is dense in $\mathfrak{M}\left(C_{b}(X, A)\right)$ and take $f \in C_{b}(X, A)$ such that $\tilde{f}$ is invertible. Then there exists an $\epsilon>0$ such that $|\widetilde{f}(x, F)|>\epsilon$ for every $(x, F) \in X \times \mathfrak{M}(A)$; hence, $\widehat{f}(G) \neq 0$ for all $G \in \mathfrak{M}\left(C_{b}(X, A)\right)$. Therefore, $f$ is invertible.

Corollary 2.9. If $X$ is a pseudocompact space, then $T(X \times \mathfrak{M}(A))$ is dense in $\mathfrak{M}\left(C_{b}(X, A)\right)$.

Proof. Suppose $f \in C_{b}(X, A)$ and $\tilde{f}$ is invertible. Then, $f$ is invertible in $C(X, A)$. Since the function $x \rightarrow\left\|f(x)^{-1}\right\|$ is continuous in $X$, then it is bounded. Therefore, $f$ is invertible in $C_{b}(X, A)$.

3. The example. We thank Vladimir Müller who orally communicated the next example to us that enables us to show that there is a completely symmetric algebra $A$ for which $T(\mathbb{N} \times \mathfrak{M}(A))$ is not dense in $\mathfrak{M}\left(C_{b}(\mathbb{N}, A)\right)$.

Let $S$ be the free commutative group with countably many generators $a_{1}, a_{2}, \ldots$ Define a function $p: S \rightarrow(0, \infty)$ by $p\left(a_{j}^{k}\right)=1$ for $k \geq 0$, $p\left(a_{j}^{k}\right)=j$ for $k<0$ and $p\left(a_{1}^{k_{1}} a_{2}^{k_{2}} \cdots a_{n}^{k_{n}}\right)=p\left(a_{1}^{k_{1}}\right) p\left(a_{2}^{k_{2}}\right) \cdots p\left(a_{n}^{k_{n}}\right)$. Then, $p$ is a positive multiplicative function.

Let $A$ be the weighted group algebra over $S$, i.e., $A$ is the set of functions $x: S \rightarrow \mathbb{C}$ satisfying that

$$
\|x\|=\sum_{s \in S}|x(s)| p(s)<\infty
$$


provided with the usual linear structure and the convolution product

$$
(x y)(s)=\sum_{t \in S} x(t) y\left(t^{-1} s\right)
$$

For each $s \in S$, let $\chi_{s}$ be the characteristic function of the singleton $\{s\}$. Then, $x=\sum_{s \in S} \alpha_{s} \chi_{s}$, with $\alpha_{s}=x(s)$, for $x \in A$. Identifying $\chi_{s}$ with $s$ in this expansion, we have

$$
\begin{aligned}
x & =\sum_{s \in S} \alpha_{s} s, \\
\|x\| & =\sum_{s \in S}\left|\alpha_{s}\right| p(s), \\
x y & =\sum_{s \in S} \sum_{t \in S} \alpha_{t} \beta_{t^{-1} s} s,
\end{aligned}
$$

if

$$
x=\sum_{s \in S} \alpha_{s} s \quad \text { and } \quad y=\sum_{s \in S} \beta_{s} s,
$$

and

$$
F(x)=\sum_{s \in S} \alpha_{s} F(s) \quad \text { for every } F \in \mathfrak{M}(A)
$$

The algebra $A$ under the involution defined by

$$
\left(\sum_{s \in S} \alpha_{s} s\right)^{*}=\sum_{s \in S} \overline{\alpha_{t}} s
$$

becomes a completely symmetric algebra.

If $B=\left\{a_{1}, a_{2}, \ldots\right\}$, then clearly $B \subset G(A)$ and $B$ is a bounded set, keeping in mind that $\left\|a_{n}\right\|=1$ for each $n$. Since $A$ is a unital commutative Banach algebra, we have that $\sigma(x)=\{F(x): F \in \mathfrak{M}(A)\}$ for each $x \in A$. From this and applying the spectral radius formula to $a_{n}$ and $a_{n}^{-1}$, we have $\left|F\left(a_{n}\right)\right|=1$ for each $n \in \mathbb{N}$ and $F \in \mathfrak{M}(A)$. Therefore, we have that $\mathfrak{M}(A)=S_{1}^{\mathbb{N}}$, associating each $F \in \mathfrak{M}(A)$ with the unique sequence $\left(e^{i \theta_{1}}, e^{i \theta_{2}}, \ldots\right)$ in the complex unit sphere $S_{1}$ such that $F\left(a_{j}\right)=e^{i \theta_{j}}$ for each $j=1,2, \ldots$.

Let us consider the algebra $C_{b}(\mathbb{N}, A)$ and the function $f \in C_{b}(\mathbb{N}, A)$ defined by $f(n)=a_{n}$ for all $n \geq 1$. Since $|\widetilde{f}(n, F)|=1$ for every $(n, F) \in \mathbb{N} \times \mathfrak{M}(A)$, the function $\widetilde{\tilde{f}}$ is invertible. Nevertheless, $f$ is not 
invertible because $(f(\mathbb{N}))^{-1}=B^{-1}$ is not bounded. Therefore, we have that $T(\mathbb{N} \times \mathfrak{M}(A))$ is not dense in $\mathfrak{M}\left(C_{b}(\mathbb{N}, A)\right)$. We point out that it can be shown that $\sigma(f)=\{z:|z| \leq 1\}$.

\section{REFERENCES}

1. J. Arhippainen, On the ideal structure of algebras of LMC-algebras valued functions, Stud. Math. 101 (1992), 311-318.

2. R.C. Buck, Continuous functions on a locally compact space, Michigan Math. J. 5 (1958), 95-104.

3. S. Dierolf, K.H. Schröder and J. Wengenroth, Characters on certain function algebras, Funct. Approx. Comm. Math. 26 (1998), 53-58.

4. W.E. Dietrich, Jr., The maximal ideal space of the topological algebra $C(X, E)$, Math. Ann. 183 (1969), 201-212.

5. Z. Ercan and S. Onal, A remark on the homomorphism on $C(X)$, Proc. Amer. Math. Soc. 133 (12) (2005), 3609-3611.

6. W. Govaerts, Homomorphisms of weighted algebras of continuous functions, Ann. Mat. Pura Appl. 116 (1978), 151-158.

7. A. Hausner, Ideals in a certain Banach algebra, Proc. Amer. Math. Soc. 8 (1957), 246-259.

8. W.J. Hery, Maximal ideal in algebras of topological algebra valued functions, Pac. J. Math. 65 (1976), 365-373.

9. L.A Kahn, Linear topological spaces of continuous vector-valued functions, Academic Publ., Ltd., 2013-DOI: 10.12732/acadpubl. 201301.

10. M.A. Naimark, Normed algebras, 1972, Wolters-Noordhoff Publishing, Groningen, The Netherlands, 1972.

11. C.E. Rickart, General theory of Banach algebras, R.E. Krieger Publishing Company, Huntington, NY, 1974 (original edition, D. Van Nostrand Reinhold, 1960).

Universidad Nacional Autónoma de México, Instituto de Matemáticas, Ciudad Universitaria, México D.F. 04510, México

Email address: hpeimbert@gmail.com

Universidad Nacional Autónoma de México, Instituto de Matemáticas, Ciudad Universitaria, México D.F. 04510, MÉxico

Email address: angel@unam.mx

Universidad nacional Autónoma de México, Facultad de Ciencias, Ciudad Universitaria, MÉxico D.F. 04510, MÉxico

Email address: alexgg577@hotmail.com 